\title{
Polaron effects in electron channels on a helium film
}

\author{
G. A. Farias and R. N. Costa Filho \\ Departamento de Física, Universidade Federal do Ceará, \\ Campus do Pici, Caixa Postal 6030, 60455-760 Fortaleza, \\ Ceará, Brazil \\ F. M. Peeters \\ Universiteit Antwerpen (UIA), Departement Natuurkunde, \\ Universiteitsplein 1, B-2610 Antwerpen, Belgium \\ Nelson Studart \\ Departamento de Física Universidade Federal de São Carlos, \\ Caixa Postal 676, 13565-905, São Carlos, São Paulo, Brazil
}

\begin{abstract}
Using the Feynman path-integral formalism we study the polaron effects in quantum wires above a liquid helium film. The electron interacts with two-dimensional (2D) surface phonons, i.e. ripplons, and is confined in one dimension (1D) by an harmonic potential. The obtained results are valid for arbitrary temperature $(T)$, electron-phonon coupling strength $(\alpha)$, and lateral confinement $\left(\omega_{0}\right)$. Analytical and numerical results are obtained for limiting cases of $T, \alpha$, and $\omega_{0}$. We found the surprising result that reducing the electron motion from 2D to quasi-1D makes the self-trapping transition more continuous.

63.20.Kr, 71.38.+i
\end{abstract}

\section{INTRODUCTION}

In recent years multi-channel electron systems have been realized over liquid helium by using one-dimensional (1D) metallic gate structures or by providing a surface distortion through a corrugated substrate in addition th the holding electric field perpendicular to the surface. A single channel was also obtained using a polymer groovel and strips on a circuit board $\mathbf{3}$ In these quasione-dimensional (Q1D) systems, the electron motion is quantized in the $z$ direction by the effective holding potential due to an external electric field and image forces coming from the substrate, and in the $y$ direction by a lateral confinement potential which can be modelled quite accurately, as in a wide class of Q1D and Q0D electron systems, by a parabolic well. 1

It is well known that the interaction of the electron with the quantized surface excitations (ripplons, in the case of a liquid surface) can be described as a polaron problem with the amazing possibility of a phasetransition like behavior between localized and delocal ized states when varying the electron-ripplon coupling. The ripplonic polaron state on the surface of helium results into a decrease of the conductivity of the electron layer. More detailed information of the polaron state can be obtained by measuring the electrop conductivity as a function of the microwave frequency. Even though the formation of the localized polaron state has been claimed, there is no conclusive interpretation about the origin of the mobility dip in some experiments and the question is still controversial.

Quite recently 1D confinement effects on surface elec- trons on bulk helium have been addressed by using the hydrodynamic model of the polaron which describes the energetics of the dimple formation on the surface of liquid helium and its transport properties.

Even though surface excitations of the liquid helium film have in general a complicated dispersion relation coming from contributions of surface tension, gravity and film thickness, in the case of thin films the ripplon spectrum has a well-defined acoustical character. ous work, 3 the $3 \mathrm{D}$ acoustical polaron was studied within the Feynman approach and it was found that, as a function of the electron-phonon coupling constant $\alpha$, the polaron undergoes a self-trapping transition at $\alpha \sim \sqrt{k_{0}}$, where $k_{0}$ is a finite Debye cutoff in phonon space, which is continuous for $k_{0}<18$ and becomes discontinuous when $k_{0}>18$. For a 2D system, a similar self-trapping transition was found at $\alpha \simeq 0.25$ which is almost $k_{0^{-}}$ independent. The transition is now continuous (discontinuous) for $k_{0}<130\left(k_{0}>130\right) .10$

In this paper, we will investigate how the polaron effect is altered when we further confine the electron system to a channel, i.e. a Q1D system. In the case of LO-phonon interaction it is well known that the polaron effect (renormalization) increases with confinement, i.e. with reduction of the dimensionality. 11.12 Here, we will study the competition between confinement and polaron effects as resulting from the interaction with acoustical phonons (ripplons). The confinement alters the available phase space of allowed $k$-vectors for virtual phonon emission and absorption and in this way the polaron effect.

The present paper is organized as follows. In Sec. II, we present the Fröhlich-type Hamiltonian, the path- 
integral Feynman formalism, 13, 14 the trial Hamiltonian used and the variational principle to derive an upper bound to the exact free energy of the system studied. Explicit analytic results in limiting cases of temperature $T$, electron-phonon coupling strength $\alpha$, and lateral confinement $\omega_{0}$ are presented in Sec. III. The numerical results are shown in Sec. IV. In the last section we will present our conclusions.

\section{HAMILTONIAN MODEL AND FREE ENERGY}

The Hamiltonian which describes an electron interacting with $2 \mathrm{D}$ phonons and subject to a lateral confinement due to an harmonic potential is given by

$$
H=\frac{\vec{p}^{2}}{2 m}+\frac{1}{2} m \omega_{0}^{2} y^{2}+\sum_{\vec{k}} \hbar \omega_{\vec{k}}\left(a_{\vec{k}}^{\dagger} a_{\vec{k}}+\frac{1}{2}\right)+H_{I},
$$

with the electron-phonon interaction given by

$$
H_{I}=\sum_{\vec{k}}\left(V_{\vec{k}} a_{\vec{k}} e^{i \vec{k} \cdot \vec{r}}+V_{\vec{k}}^{*} a_{\vec{k}}^{\dagger} e^{-i \vec{k} \cdot \vec{r}}\right)
$$

where $\vec{r}(\vec{p})$ is the $2 \mathrm{D}$ position (momentum) operator of the electron with mass $m, V_{\vec{k}}$ is the electron-phonon strength and $a_{\vec{k}}^{\dagger}\left(a_{\vec{k}}\right)$ are the creation (annihilation) operators of the phonons with wave vector $\vec{k}$, and frequency $\omega_{\vec{k}}$. The electron is laterally confined by an harmonic potential with frequency $\omega_{0}$.

The Helmholtz free energy is given by

$$
F=-\frac{1}{\beta} \log Z,
$$

with

$$
Z=\operatorname{Tr} e^{-\beta H},
$$

the partition function. The free energy, Eq. (3), and the partition function, Eq. (4), contain the contribution of the free phonons, $F_{p h}$. Here, we will shift the free energy $F$ by this constant contribution $F_{p h}$. Using the path-iptegral formalism, the trace of the partition function 13.15 .16 can be written as

$$
Z=\int d \vec{r} \int D \vec{r}(u) \exp \{S[\vec{r}(u)]\} \delta(\vec{r}(\beta)-\vec{r}) \delta(\vec{r}(0)-\vec{r}),
$$

where $\int D \vec{r}$ denotes the integral over all possible electron paths, $\beta=1 / k_{B} T$ is the inverse temperature, and $u$ is related to the real time variable $t$ as $u=i t$. The action $S[\vec{r}]$ in Eq. (5) is obtained after the exact elimination of the phonon coordinates and is given by 15

$$
S=S_{e}+S_{I},
$$

with

$$
S_{e}=-\frac{m}{2} \int_{0}^{\beta} d t\left(\dot{x}(t)^{2}+\dot{y}(t)^{2}\right)-\frac{1}{2} m \omega_{0}^{2} \int_{0}^{\beta} d t y(t)^{2},
$$

$$
S_{I}=\sum_{\vec{k}}\left|V_{\vec{k}}\right|^{2} \int_{0}^{\beta} d t \int_{0}^{\beta} d s G_{\omega_{k}}(t-s) e^{\imath \vec{k} \cdot(\vec{r}(t)-\vec{r}(s))},
$$

where

$$
G_{\omega}(t)=\frac{1}{2}\left[(1+n(\omega)) e^{-\hbar \omega|t|}++n(\omega) e^{\hbar \omega|t|}\right]
$$

is the phonon Green's function, and $n(\omega)=\left(e^{\beta \hbar \omega}-1\right)^{-1}$ the number of phonons with frequency $\omega$.

The path-integral in Eq. (5), can not be evaluated exactly. We follow Feynman's polaron theory 13 and introduce a trial action $S_{0}$, such that: i) the path integral with this action can be done exactly, and (ii) it approximates the original action $S$ as close as possible. The free energy associated with this trial action is given by

$$
\begin{aligned}
Z_{0}=e^{-\beta F_{0}}= & \int d \vec{r} \int D \vec{r}(u) \exp \left\{S_{0}[\vec{r}(u)]\right\} \times \\
& \delta(\vec{r}(\beta)-\vec{r}) \delta(\vec{r}(0)-\vec{r}),
\end{aligned}
$$

and we define the expectation value of any functional $A[\vec{r}]$ with respect to $S_{0}$ by

$$
\begin{aligned}
\langle A[\vec{r}]\rangle_{0}=\frac{1}{Z_{0}}= & \int d \vec{r} \int D \vec{r}(u) \exp \left\{S_{0}[\vec{r}(u)]\right\} \times \\
& =[\vec{r}(u)] \delta(\vec{r}(\beta)-\vec{r}) \delta(\vec{r}(0)-\vec{r}) .
\end{aligned}
$$

Using this trial action, the partition function of the real system, Eq. (5), can be written as

$$
Z=Z_{0}\left\langle\exp \left(S-S_{0}\right)\right\rangle_{0}
$$

If the actions $S$ and $S_{0}$ are real, as is the case for our problem, the convexity property of the function $\exp (x)$ can be applied from which we find the inequality

$$
\left\langle\exp \left(S-S_{0}\right)\right\rangle_{0} \geq \exp \left(\left\langle S-S_{0}\right\rangle_{0}\right) .
$$

leading to a variational principle for the free energy

$$
F \leq F_{0}-\frac{1}{\beta}\left\langle S-S_{0}\right\rangle_{0} .
$$

We propose a trial action derivable from the trial Hermitian Hamiltonian

$$
H_{0}=\frac{p_{x}^{2}}{2 m}+\frac{p_{X}^{2}}{2 M}+\frac{1}{2} K(x-X)^{2}+\frac{p_{y}^{2}}{2 m}+\frac{1}{2} m \omega^{\prime 2} y^{2},
$$

where $X\left(p_{X}\right)$ is the $1 \mathrm{D}$ position (momentum) of the fictitious particle of mass $M, K$ is the strength of the 
harmonic potential and $\omega^{\prime}$ is the oscillation frequency associated with the lateral confinement of the electron in the $y$ direction. The first three terms in Eq. (12) corre spond to the 1-D Feynman polaron in the $x$ direction. 17 The last two are associated to the confined motion and the variables $M, K$ and $\omega^{\prime}$ are variational parameters.

Using the same procedure as for the original Hamiltonian, Eq. (1), we eliminate the coordinates of the fictitious particle, and obtain the trial action

$$
\begin{aligned}
S_{0}= & -\frac{m}{2} \int_{0}^{\beta} d t\left(\dot{x}(t)^{2}+\dot{y}(t)^{2}\right)-\frac{1}{2} m \omega^{\prime 2} \int_{0}^{\beta} d t y(t)^{2} \\
& -\frac{\hbar^{2} K^{2}}{4 M \omega} \int_{0}^{\beta} d t \int_{0}^{\beta} d s G_{\omega}(t-s)(x(t)-x(s))^{2}
\end{aligned}
$$

with $\omega=\sqrt{K / M}$ and $v^{2}=K[(M+m) / M m]$.

Following the same approach used by Peeters and Devreese 18 , from Eqs. (6a) and (13), the Feynman variational principle for the free energy of our system, Eq. (11), can be written as

$$
F \leq F_{F}-F_{R}-\frac{1}{\beta}\left\langle S-S_{0}\right\rangle_{0},
$$

where $F_{F}$ is the free energy of the trial Hamiltonian, Eq. (12), given by

$$
\begin{aligned}
F_{F}= & -\frac{1}{\beta} \log \left[L_{x} \frac{v}{w} \frac{1}{\sqrt{2 \pi \beta}}\right] \\
& -\frac{1}{\beta} \log \left[\left\{\frac{1}{2 \sinh \frac{1}{2} \beta v}\right\}\left\{\frac{1}{2 \sinh \frac{1}{2} \beta \omega^{\prime}}\right\}\right]
\end{aligned}
$$

$F_{R}$ is the free energy of the fictitious particle without the interaction term, given by

$$
F_{R}=-\frac{1}{\beta} \log \left[\frac{1}{2 \sinh \frac{1}{2} \beta \omega}\right]
$$

and

$$
\begin{aligned}
\left\langle S-S_{0}\right\rangle_{0} & =\frac{1}{2} m\left(\omega^{\prime 2}-\omega_{0}^{2}\right) \int_{0}^{\beta} d t\left\langle y(t)^{2}\right\rangle_{0}+\sum_{\vec{k}}\left|V_{\vec{k}}\right|^{2} \times \\
& \int_{0}^{\beta} d t \int_{0}^{\beta} d s G_{\omega_{k}}(t-s)\left\langle e^{\imath \vec{k} \cdot(\vec{r}(t)-\vec{r}(s))}\right\rangle_{0} \quad(15 \mathrm{c}) \\
& -\frac{\hbar m}{4} \omega\left(v^{2}-\omega^{2}\right) \int_{0}^{\beta} d t \int_{0}^{\beta} d s \times \\
& G_{\omega}(t-s)\left\langle(x(t)-x(s))^{2}\right\rangle .
\end{aligned}
$$

Defining

$$
\left\langle e^{\imath \vec{k} \cdot(\vec{r}(t)-\vec{r}(s))}\right\rangle_{0}=A_{x}\left(k_{x}, t-s\right) A_{y}\left(k_{y}, t-s\right),
$$

where

$$
A_{\xi}\left(k_{\xi}, t-s\right)=\left\langle e^{\imath k_{\xi}(\xi(t)-\xi(s))}\right\rangle_{0}
$$

with $\xi=x, y$, we have that

$$
\left\langle(\xi(t)-\xi(s))^{2}\right\rangle_{0}=-\frac{\partial^{2}}{\partial k_{\xi}^{2}} A_{\xi}\left(k_{\xi}, t-s\right) .
$$

The three averages in Eq. (15c) can be calculated in an analogous way as done by Osaka 15 and Peeters and Devresee 18 such that we obtain

$$
\begin{aligned}
& \frac{1}{m}\left\langle y(t)^{2}\right\rangle_{0}=\frac{1}{2 \omega^{\prime}} \operatorname{coth} \frac{\beta \omega^{\prime}}{2} \\
& A_{\xi}\left(k_{\xi}, t-s\right)=e^{-k_{\xi}^{2} D_{\xi}(t-s)}
\end{aligned}
$$

with

$$
\begin{aligned}
D_{x}(t)= & \frac{|t| \omega^{2}}{2 v^{2}}\left(1-\frac{|t|}{\beta}\right) \\
& -\frac{v^{2}-\omega^{2}}{2 v^{3}}\left(1-e^{-v|t|}-4 n(v) \sinh ^{2} \frac{v t}{2}\right),
\end{aligned}
$$

and $D_{y}(t)$ is obtained from the above expression by taking $\omega \rightarrow 0$ and $v \rightarrow \omega^{\prime}$, giving

$$
D_{y}(t)=\frac{1}{2 \omega^{\prime}}\left(1-e^{-\omega^{\prime}|t|}-4 n(v) \sinh ^{2} \frac{\omega^{\prime} t}{2}\right) .
$$

Substituting Eqs. 115a)- 21b in Eq. 114, we finally obtain an upper bound $F^{v}$ for the exact free energy of our system, given by

$$
\begin{aligned}
F^{v} & =\frac{1}{4 v}\left(v^{2}-\omega^{2}\right)\left(\operatorname{coth} \frac{\beta v}{2}-\frac{2}{\beta v}\right)-\frac{1}{\beta} \log \left[\frac{v}{w} \frac{L_{x}}{\sqrt{2 \pi \beta}}\right] \\
& -\frac{1}{\beta} \log \left[\left(\frac{\sinh \frac{1}{2} \beta \omega}{\sinh \frac{1}{2} \beta v}\right)\left\{\frac{1}{2 \sinh \frac{1}{2} \beta \omega^{\prime}}\right\}\right]-\frac{1}{4 \omega^{\prime}} \times \\
& \left(\omega^{\prime 2}-\omega_{0}^{2}\right) \operatorname{coth} \frac{\beta \omega^{\prime}}{2}-\sum_{\vec{k}}\left|V_{\vec{k}}\right|^{2}\left[1+n\left(\omega_{\vec{k}}\right)\right] \times \\
& \int_{0}^{\beta} d u e^{-\omega_{\vec{k}} u} e^{-k_{x}{ }^{2} D_{x}(u)} e^{-k_{y}{ }^{2} D_{y}(u)},
\end{aligned}
$$

which is subject to the minimization conditions

$$
\frac{\partial F^{v}}{\partial v}=\frac{\partial F^{v}}{\partial \omega}=\frac{\partial F^{v}}{\partial \omega^{\prime}}=0 .
$$

\section{GROUND-STATE ENERGY AND LIMITING CASES}

The result obtained in Eq. (22) is general and can be used to calculate all thermodynamic quantities, like e.g. specific heat, entropy, internal energy, etc. In the zero-temperature limit, the free energy $F^{v}$ reduces to the polaron ground-state energy $E_{0}{ }^{v}$, given by

$$
\begin{gathered}
E_{0} v=\lim _{\beta \rightarrow \infty} F^{v}=\frac{1}{2} \omega^{\prime}-\frac{\left(\omega^{\prime 2}-\omega_{0}^{2}\right)}{4 \omega^{\prime}}+\frac{1}{4 v}(v-\omega)^{2}- \\
\sum_{\vec{k}}\left|V_{\vec{k}}\right|^{2} \int_{0}^{\infty} d u e^{-\omega_{\vec{k}} u} e^{-k_{x}{ }^{2} D_{x}{ }^{0}(u)} e^{-k_{y}{ }^{2} D_{y}{ }^{0}(u)},
\end{gathered}
$$


with

$$
D_{x}{ }^{0}(u)=\frac{\omega^{2}}{2 v^{2}} u+\frac{v^{2}-\omega^{2}}{2 v^{3}}\left(1-e^{-v u}\right),
$$

and $D_{y}(t)$ is obtained from the above expression by taking $\omega \rightarrow 0$ and $v \rightarrow \omega^{\prime}$, giving

$$
D_{y}{ }^{0}(u)=\frac{1}{2 \omega^{\prime}}\left(1-e^{-\omega^{\prime} u}\right) .
$$

In Eq. (24) the first term corresponds to the zero-point energy of the harmonic oscillator with frequency $\omega^{\prime}$, the second one is due to the interaction between the lateral confinement and the fictitious oscillator with frequencies $\omega_{0}$ and $\omega^{\prime}$, respectively. The third term is due to the Feynman polaron with eigenfrequency $v$ and the fictitious particle with frequency $w$ (i.e. $m \rightarrow \infty$ ). The last term is associated with the electron-phonon interaction.

If the $y$-confinement is much larger than the phonon effects, one can take the limit $\omega^{\prime} \approx \omega_{0}$. Also, if in this limit the phonon cloud surrounding the electron is very small, we can assume $v \approx w$. Within these approximations, the polaron ground-state energy $E_{0}{ }^{v}$, Eq. 244, is given by

$$
\begin{aligned}
E_{0}{ }^{\text {conf }}= & \frac{1}{2} \omega_{0}-\sum_{\vec{k}}\left|V_{\vec{k}}\right|^{2} \times \\
& \int_{0}^{\infty} d u e^{-\omega_{\vec{k}} u} e^{-k_{x}{ }^{2} u / 2} e^{-k_{y}{ }^{2}\left(1-e^{-\omega_{0} u}\right) / 2 \omega_{0}},
\end{aligned}
$$

which equals the result from second-order perturbation theory.

The first term in $E_{0}{ }^{c o n f}$ is the zero-point energy of the harmonic oscillator, the second exponential term under the integral is associate with the free motion along the $x$ direction, and the last exponential under the integral is due to the oscillator motion in the confinement direction containing all confinement levels. Expanding the last exponential term under the integral of $E_{0}$ conf , we can integrate out the $u$ variable resulting in

$$
\begin{aligned}
E_{0}{ }^{c o n f}= & \frac{1}{2} \omega_{0}-\sum_{\vec{k}}\left|V_{\vec{k}}\right|^{2} \times \\
& \sum_{n=0}^{\infty} \frac{1}{n !}\left(\frac{k_{y}{ }^{2}}{2 \omega_{0}}\right)^{n} \frac{e^{-k_{y}{ }^{2} / 2 \omega_{0}}}{\omega_{k}+k_{x}^{2} / 2+n \omega_{0}},
\end{aligned}
$$

which allows a perturbation analysis in terms of diagrams.

We now consider the limiting cases of weak and strong coupling. For weak coupling, we have the following conditions: $\alpha \gg 0.5, v \approx w$, and $\omega^{\prime} \rightarrow 0$. Therefore, expanding the exponentials in the $D_{x, y}$ terms, we obtain $D_{x}(u) \approx u / 2$ and $D_{y}(u) \approx u / 2$, resulting in $f(k, u) \simeq \exp \left(-k^{2} u / 2\right)$. In this case, the integral

$$
\begin{aligned}
B & =\alpha \int_{0}^{k_{0}} d k k^{2} \int_{0}^{\infty} d u e^{-k u} f(k, u) \\
& \simeq 2 \alpha k_{0}\left[1-\frac{2}{k_{0}} \ln \left(1+\frac{k_{0}}{2}\right)\right],
\end{aligned}
$$

and the polaron energy can be written as

$$
E_{p o l}=\frac{1}{4}\left(\frac{\omega_{0}^{2}}{\omega^{\prime}}\right)-B .
$$

In the absence of confinement $\left(\omega_{0}=0\right)$, even when $\omega^{\prime}$ is too small, $E_{\text {pol }}=-B$. For $\omega_{0}>1$, the polaron energy increases fast.

In the strong limit, $\alpha \gg 0.5$, we have the conditions: $v \gg w$, and $\omega^{\prime} \rightarrow v$. Therefore, as before, expanding the exponentials in the $D_{x, y}$ terms we obtain now $D_{x}(u) \approx 1 / 2 v$ and $D_{y}(u) \approx 1 / 2 \omega^{\prime}$, resulting in $f(k, u) \simeq \exp \left(-k^{2} / 2 v\right)$. In this case, the integral

$$
\begin{aligned}
B & =\alpha \int_{0}^{k_{0}} d k k^{2} \int_{0}^{\infty} d u e^{-k u} f(k, u) \\
& \simeq-\alpha v\left(e^{-k_{0}^{2} / 2 v}-1\right)
\end{aligned}
$$

and the energy is given as

$$
E_{p o l}=\frac{v}{2}-\frac{\omega_{0}^{2}}{4 v}+\alpha v\left(e^{-k_{0}^{2} / 2 v}-1\right) .
$$

One can see that the energy decreases when we increase the lateral confinement.

\section{NUMERICAL RESULTS}

In this section we present the numerical results for the zero-temperature limit of the free energy $F^{v}$, that is, the confinement polaron ground-state energy $E_{0}{ }^{v}$, by minimizing Eq.(24), and considering the electron interacting with longitudinal surface acoustic phonons (ripplons). For these excitations, the dispersion relation is given by

$$
\omega_{\vec{k}}=s|\vec{k}|,
$$

where $s$ is the velocity of sound, and the Fourier transform of the electron-phonon interaction

$$
\left|V_{\vec{k}}\right|^{2} \propto \alpha k
$$

describes both 2D phonons and ripplons 10 , where $\alpha$ is the dimensionless electron-phonon coupling constant which depends on the deformation potential. In our calculation the sum over the phonon wave vectors $\sum_{\vec{k}}$ will be replaced by the integral $A /(2 \pi)^{2} \int d \vec{k}$, which is cutoff at $k_{0}$, the Debye critical wave vector in phonon space which simulates the discreteness of the lattice, $k_{0} \sim 1 / a$, with $a$ the lattice constant, and corresponds to the capillary constant $k_{c} \sim 1 / d^{2}$, where $d$ is the film thickness in case of liquid helium. Hereafter, we use dimensionless units and express the energy in units of $m s^{2}$ and the length in units of $\hbar / m s$. Substituting Eqs. (32a)-(32b) in Eq. (24), and integrating out the angular coordinate of $\vec{k}$ we obtain 


$$
\begin{aligned}
E_{0}{ }^{v}= & \frac{1}{2} \omega^{\prime}-\frac{1}{4 \omega^{\prime}}\left(\omega^{\prime 2}-\omega_{0}^{2}\right)+\frac{1}{4 v}(v-\omega)^{2} \\
& -\alpha \int_{0}^{k_{0}} d k k^{2} \int_{0}^{\infty} d u e^{-k u} f(k, u),
\end{aligned}
$$

with

$$
\begin{aligned}
f(k, u)= & e^{-\frac{1}{2} k_{x}{ }^{2}\left(D_{x}{ }^{0}(u)+D_{y}{ }^{0}(u)\right)} \times \\
& I_{0}\left(\frac{k_{x}{ }^{2}}{2}\left|D_{x}{ }^{0}(u)-D_{y}{ }^{0}(u)\right|\right) .
\end{aligned}
$$

The results for the energy as a function of $\alpha$ are shown in Fig. 1, for different values of the Debye cutoff $k_{0}$ and the lateral confinement frequency $\omega_{0}$. The figure shows that the slope of the polaron energy varies at $\alpha \approx 0.51$. This value of $\alpha$ is approximatelly twice the value obtained in Ref.10, even when we take the limit $\omega_{0} \rightarrow 0$. This difference can be understood as due to the fact that the translational invariance in the $y$ direction is not taken into account in our model Hamiltonian, i.e, we are considering a Q1D system.

As can be seen, the lateral confinement increases the energy and this is more significant for small values of $\alpha$. In order to analyze the effects of the lateral confinement on the self-trapping transition, Fig. 2 shows the first derivative of the ground-state energy as a function of $\alpha$ for two values of $k_{0}$. We observe, in Fig. 2(a), that the lateral confinement, $\omega_{0}=20$, smoothens the curve compared with the one where $\omega_{0}=0$. Considering $k_{0}=150$, Fig. 2(b) shows the existence of a discontinuity in the first derivative corresponding to a first-order transition in the case where $\omega_{0}=0$. This is not observed for $\omega_{0}=20$. These facts are best visualized in the second derivative of the ground-state energy as function of the coupling constant presented in Fig. 3.

In Fig. 4 , the phase diagram $\left(k_{0}, \omega_{0}\right)$ for the $2 \mathrm{D}$ acoustical polaron is depicted for $\alpha=0.515$ where the transition occurs. For each $\omega_{0}$, the value of $k_{0}$ determines the self-trapped transition and the curve bounds two regions. In the upper region, the transition is discontinuous, while in the below region it is continuous for this $\alpha$-value. In the latter region it is possible to find a discontinous selftrapping transition when $\alpha>0.515$.

In Fig. 5 we present the Feynman polaron mass $M=$ $(v / w)^{2}$ as a function of $\alpha$, for different values of the Debye cutoff $k_{0}$ and the lateral confinement frequency $\omega_{0}$. The figure shows clearly the transition from the weak- to strong-coupling regime. It also shows that for $k_{0}<70$ the transition is continuous. Again, it is worth to mention that, for $\omega_{0}=0.0$, the obtained here is different from the the $2 \mathrm{D}$ polaron. 10 Once more, this difference is due to the fact that the translational invariance in the $y$ direction is not taken into account in our model Hamiltonian.

We also observe that the effects of the lateral confinement on the polaron mass are more significant in the region of small $\alpha$, i.e. the non self-trapped region, and the transition from low to large polaron mass is now smooth.
In Fig. 6 we show the ground-state energy shift $\Delta E=$ $E\left(\omega_{0}=0\right)-E\left(\omega_{0}\right)$ as a function of $\omega_{0}$, for different values of $k_{0}$ and a fixed value of $\alpha$. The lateral confinement increases the binding energy and we do not see any abrupt change in the energy values. This fact can be understood from Eq. (27). This result shows that, when $\omega_{0}$ increases, the zero-point energy increases more than the terms corresponding to the free motion along the $x$ direction, and the one associated to the oscillator motion in the confinement direction, that contain all the confinement levels. Although the ground-state energy does not present an abrupt change with the confinement this fact is observed in the Feynman polaron mass. In Fig. 7 we present the Feynman polaron mass $M=(v / w)^{2}$ as a function of $\omega_{0}$, for different values of $k_{0}$ and a fixed value of $\alpha$. As can be seen, the polaron mass increases with the lateral confinement and can change dramatically for large values of $k_{0}$ where it exhibits a discontinuous behavior.

\section{CONCLUSIONS}

In this work we applied the Feynman path-integral method to study the effects of lateral confinement on the ground-state properties of the $2 \mathrm{D}$ acoustical polaron which can be formed in Q1D electron systems over a helium film. We evaluated the ground-state energy and the polaron mass as a function of the electron-phonon coupling $\alpha$ and we analyzed the effect of lateral confinement on these properties. We determined the phase diagram for polaron formation and found the intriguing result that the self-trapping transition becomes more continuous when the electron motion is reduced from $2 \mathrm{D}$ to a Q1D system.

\section{ACKNOWLEDGMENTS}

G.A.F, R.N.C.F and N.S are supported by the Conselho Nacional de Desenvolvimento Científico e Tecnológico $(\mathrm{CNPq})$ and F.M.P is sponsored by the Belgian National Science Foundation and the EC-project: HPRN-CT-2000-00157. N.S is grateful to Fundação de Amparo à Pesquisa do Estado de São Paulo (FAPESP) for a research grant.

${ }^{1}$ N. Studart and S. S. Sokolov, in Two-Dimensional Electron Systems in Helium and Other Substrates, edited by E. Y. Andrei, Kluwer, Dordrecht (1997) and references therein.

${ }^{2}$ O. I. Kirichek, Yu. P. Monarkha, Yu. Z. Kovdrya, and V. N. Grigorév, Fiz. Nizk, Temp. 19, 458 (1993) [Low Temp. Phys. 19, 323 (1993)]. 
${ }^{3}$ A. M. C. Valkering and R. W. van der Heijden, Physica B 249-251, 652 (1998).

${ }^{4}$ S. S. Sokolov, G. Q. Hai, and N. Studart, Phys. Rev. B 51, 2640 (1995).

${ }^{5}$ S. Jackson and P. M. Platzman, Phys. Rev. B 24, 499 (1981); O. Hipólito, G. A. Farias, and N. Studart, Surf. Sci. 113, 394 (1982); M. Saitoh, J. Phys. C: Solid State Phys. 16, 6695 (1983); S. A. Jackson and F. M. Peeters, Phys. Rev. B 30, 4196 (1984); F. M. Peeters and S. A. Jackson, Phys. Rev. B 31, 7098 (1985).

${ }^{6}$ See for instance, A. J. Dahm in Two-Dimensional Electron Systems in Helium and Other Substrates, edited by E. Y. Andrei, Kluwer, Dordrecht (1997).

${ }^{7}$ E. Y. Andrei, Phys. Rev. Lett. 52, 1449 (1984); F. F. Mende, Yu. Z. Kovdrya, and V. A. Nikolaenko, Fiz. Nizk, Temp. 11, 646 (1985) [Low Temp. Phys. 11, 355 (1985)]; F.M. Peeters and S.A. Jackson, Phys. Rev. B 34, 1539 (1986); X. L. Hu, Y. Carmi, and A. J. Dahm, J. Low Temp. Phys. 89, 625 (1992); O. Tress, Yu. P. Monarkha, F. C. Penning, H. Bluyssen, and P. Wyder, Phys. Rev. Lett. 77, 2511 (1996); ibid. 82, 2005 (1999); N. A. Rubin and A. J. Dahm, Phys. Rev. Lett. 77, 2004 (1999).

${ }^{8}$ S. S. Sokolov, A. C. A. Ramos, and N. Studart, J. Phys.: Condens. Matter 12, 7341 (2000).

${ }^{9}$ F. M. Peeters and J. T. Devreese, Phys. Rev. B 32, 3515 (1985).

${ }^{10}$ G. A. Farias, W. B. da Costa, and F. M. Peeters, Phys. Rev. B 54, 12835 (1996)

${ }^{11}$ F. M. Peeters, X.-G. Wu, and J. T. Devreese, Phys. Rev. B 33, 3926 (1986).

${ }^{12}$ M. H. Degani and G. A. Farias, Phys. Rev. B 42, 11950 (1990).

${ }^{13}$ R. P. Feynman, Phys. Rev. 97, 660 (1955).

${ }^{14}$ See also R. P. Feynman, Statistical Mechanics: A Set of Lectures (Benjamin, Reading, MA, 1972) and R. P. Feynman and A. R. Hibbs, Quantum Mechanics and Path Integrals, (McGraw-Hill, New York, 1965).

15 Y. Osaka, Prog. Theor. Phys. 22, 437 (1957).

16 T. D. Schultz, in Polarons and Excitons, edited by C. G. Kuper and G. P. Whithfield (Oliver and Boyd, Edinburg, 1963), p. 71.

${ }^{17}$ M. H. Degani, O. Hipólito, R. Lobo, and G. A. Farias, J. Phys. C: Solid State Phys. 19, 2919 (1986).

${ }^{18}$ F. M. Peeters and J. T. Devreese, Phys. Rev. B 23, 1935 (1981); 25, 7281 (1982); 25, 7302 (1982).

\section{FIGURES}

Fig. 1. Ground-state energy of the 2D acoustical polaron as a function of the electron-phonon coupling constant $\alpha$ for different values of $k_{0}, \omega_{0}=20$ (dash-dotted lines) and $\omega_{0}=0$ (solid lines).

Fig. 2. First derivative of the ground-state energy of the $2 \mathrm{D}$ acoustical polaron as a function of the electronphonon coupling constant $\alpha$ for: a) $k_{0}=50$, b) $k_{0}=150$, in both cases for $\omega_{0}=0$ (solid lines) and $\omega_{0}=20$ (dashdotted lines).

Fig. 3. Second derivative of the ground-state energy of the $2 \mathrm{D}$ acoustical polaron as a function of the electronphonon coupling constant $\alpha$ for: a) $k_{0}=50$, and b) $k_{0}=$ 150 , in both cases for $\omega_{0}=0$ (solid lines) and $\omega_{0}=20$ (dash-dotted lines).

Fig. 4. Phase diagram $\left(k_{0}, \omega_{0}\right)$ for the self-trapping transition of the acoustical polaron at $\alpha=0.515$.

Fig. 5. Feynman polaron mass $M=(v / w)^{2}$ as a function of the electron-phonon coupling constant $\alpha$ for different values of $k_{0}$ and $\omega_{0}$.

Fig. 6. The ground-state energy shift of the polaron, $\Delta E=E\left(\omega_{0}=0\right)-E\left(\omega_{0}\right)$ as a function of the lateral confinement frequency $\omega_{0}$ for $\alpha=0.515$, considering: $k_{0}=50$ the solid line, $k_{0}=100$ the dashed line, and $k_{0}=100$ the dash-dotted line.

Fig. 7. Polaron mass as a function of the lateral confinement frequency $\omega_{0}$ for the same parameters as in Fig. 6 . 


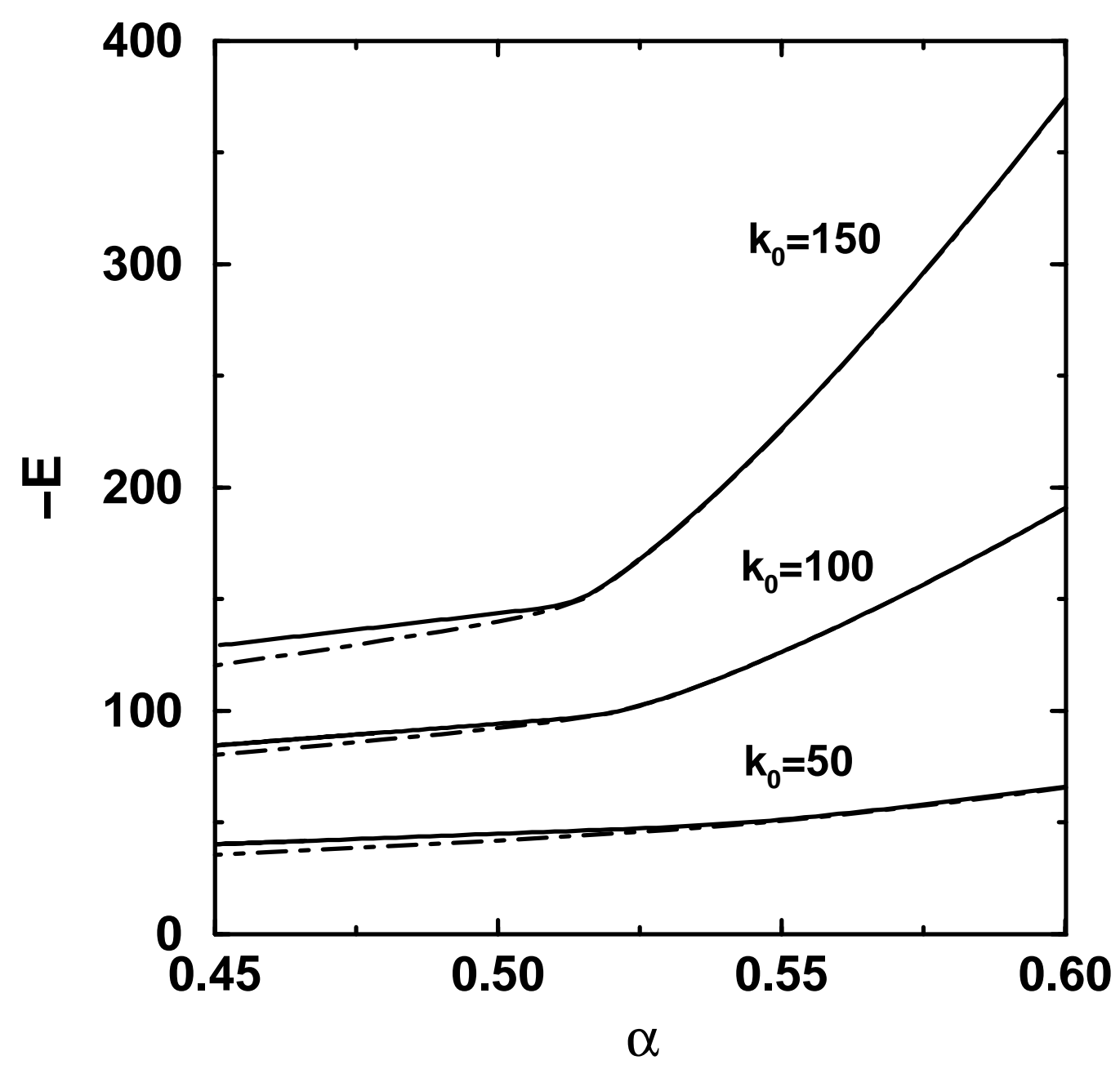




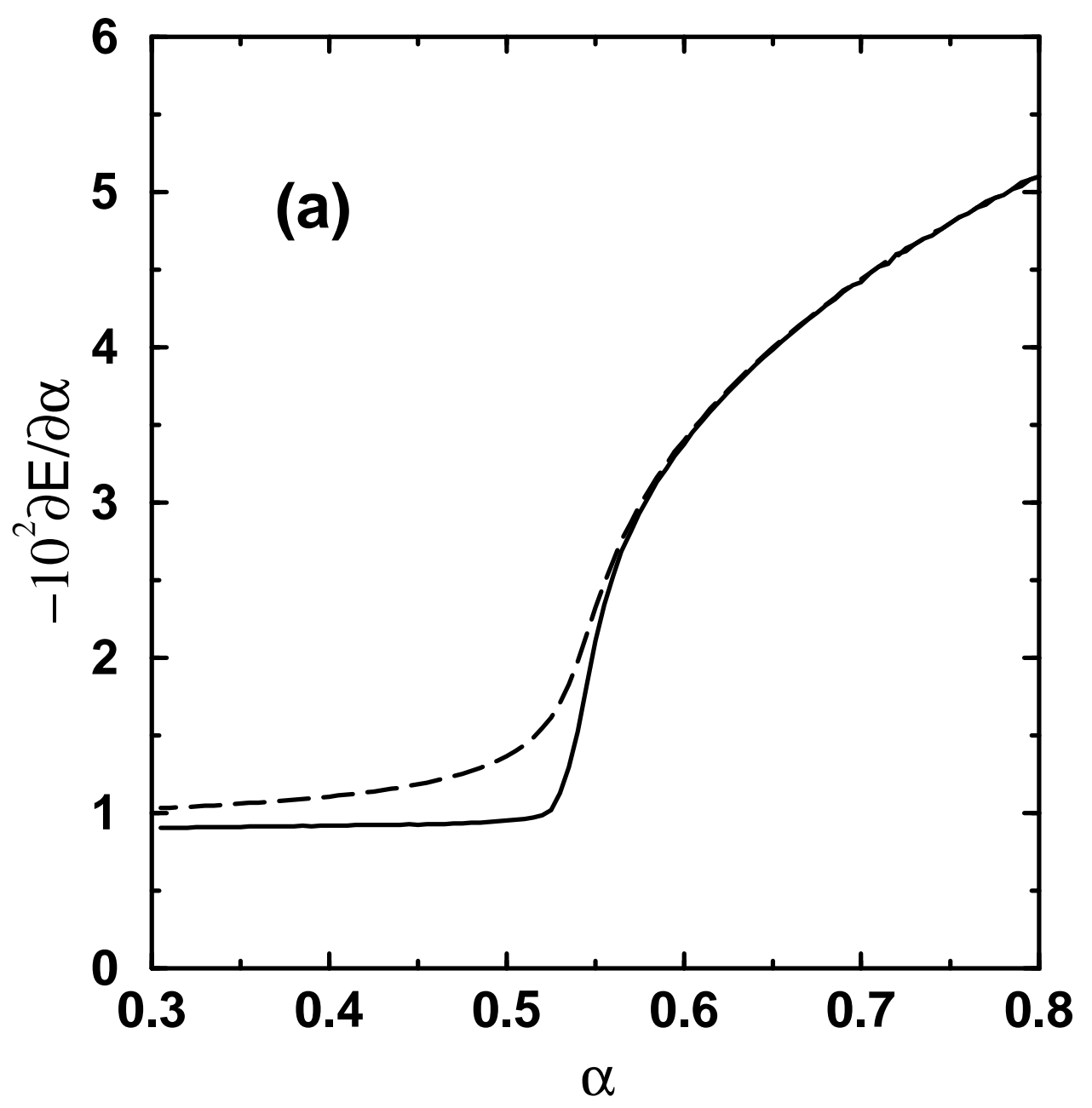




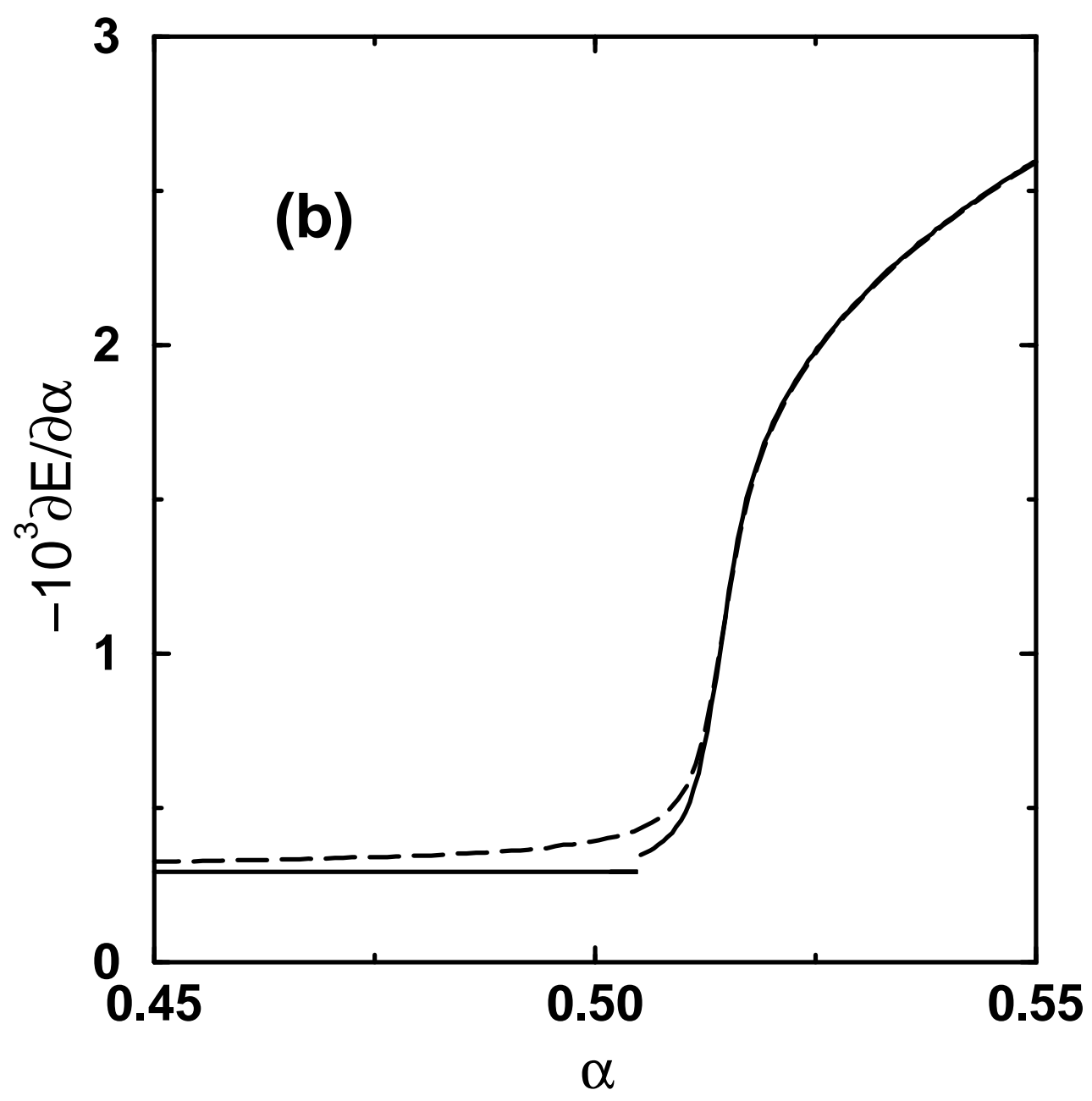




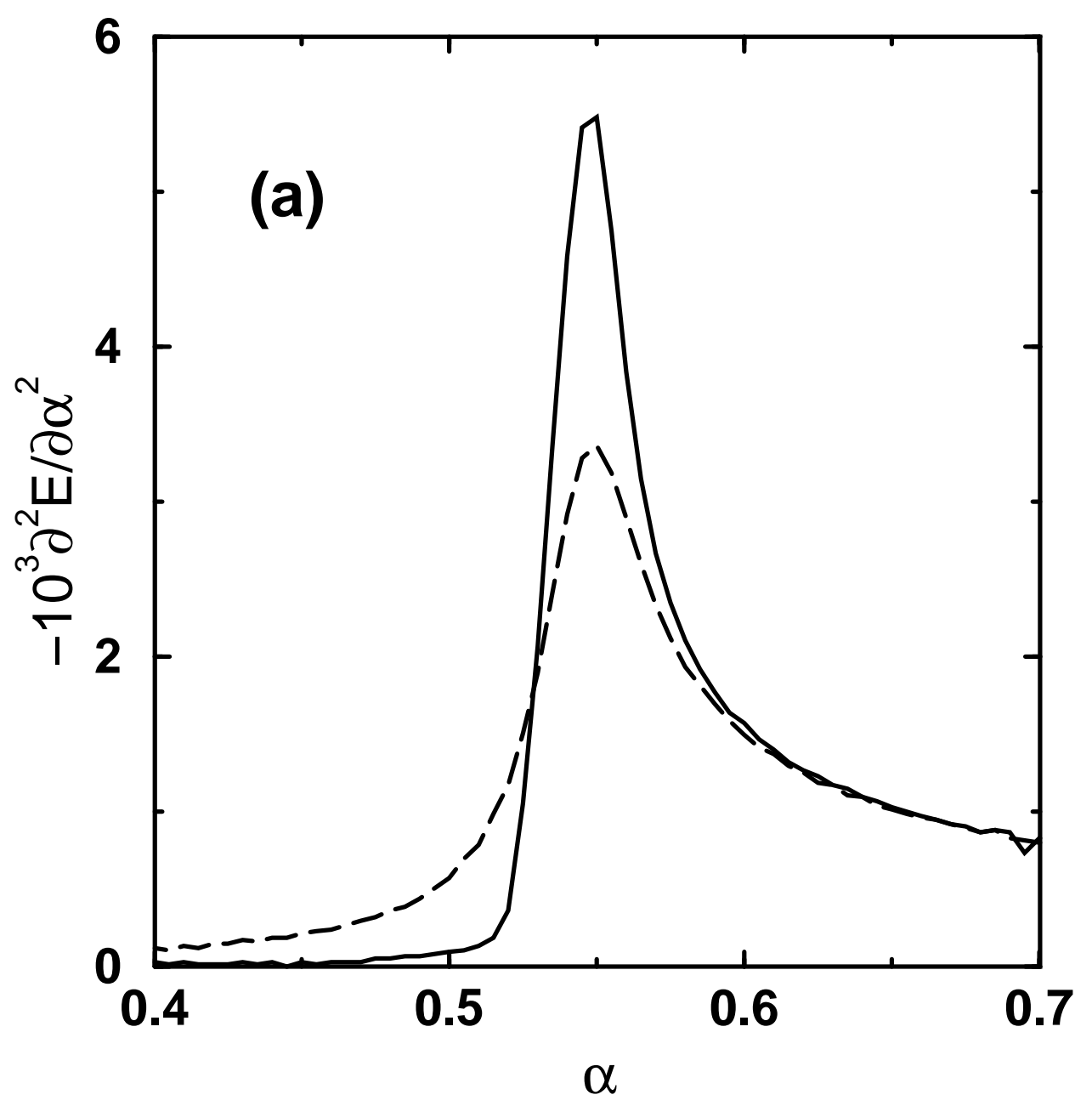




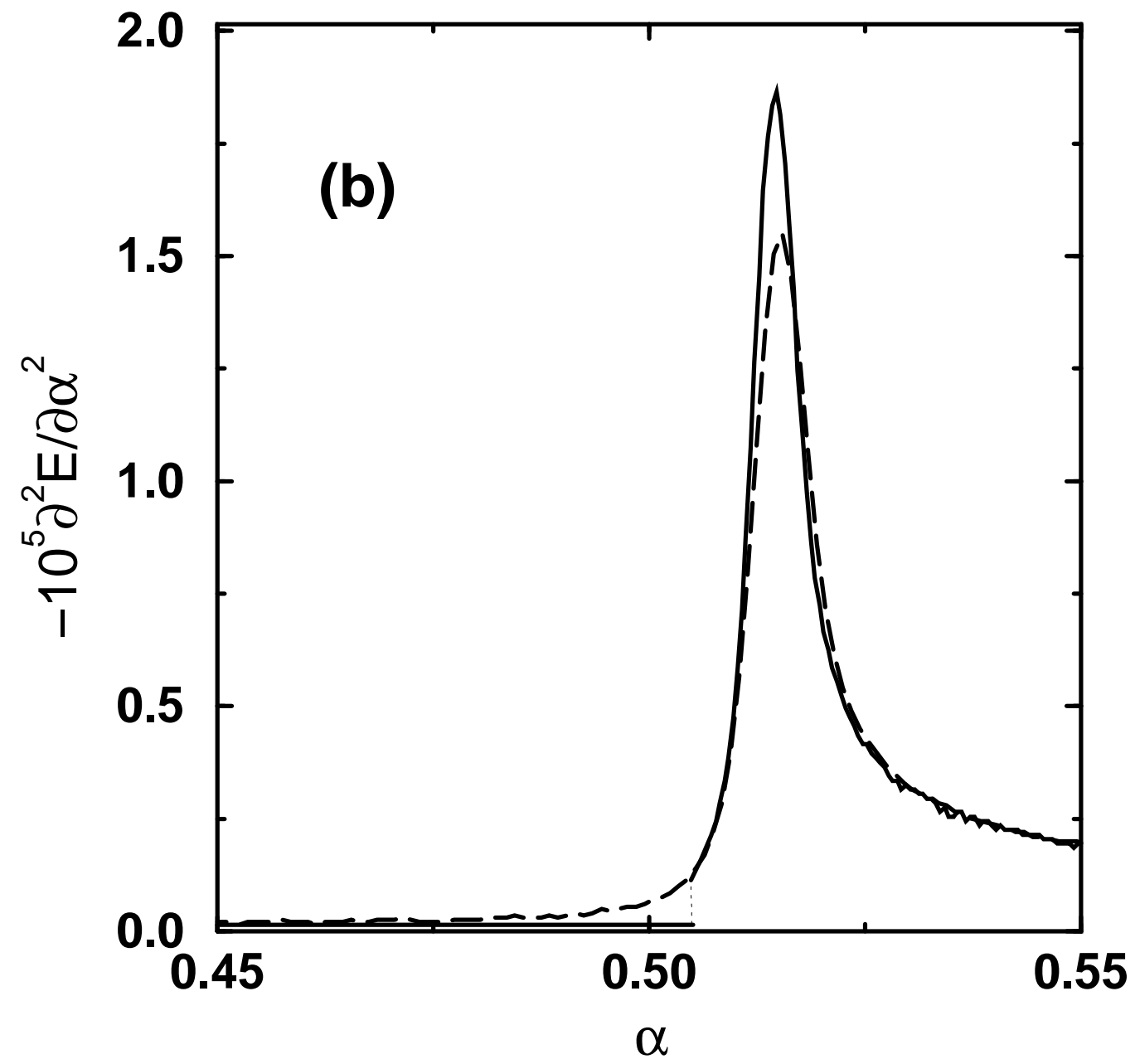




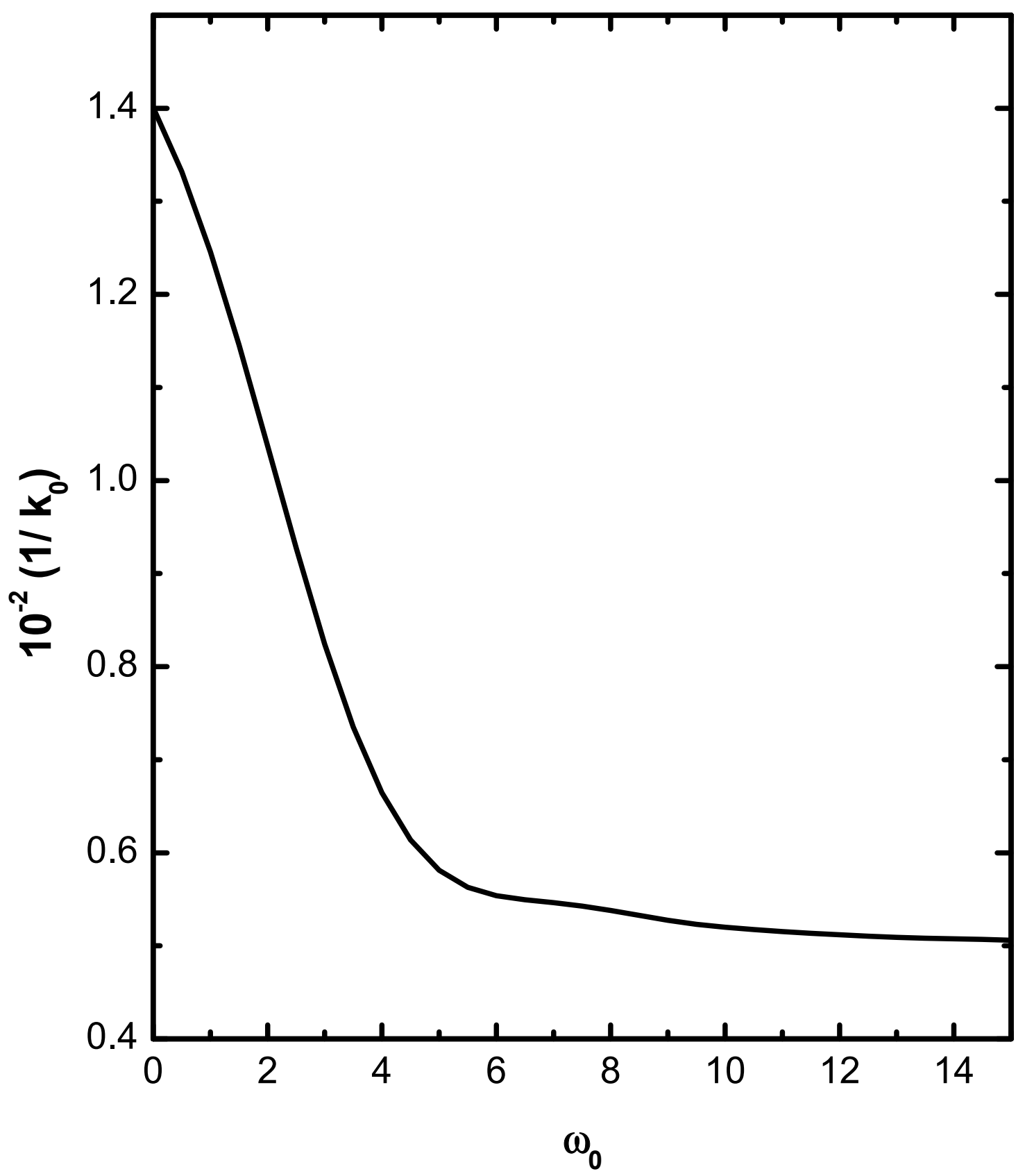




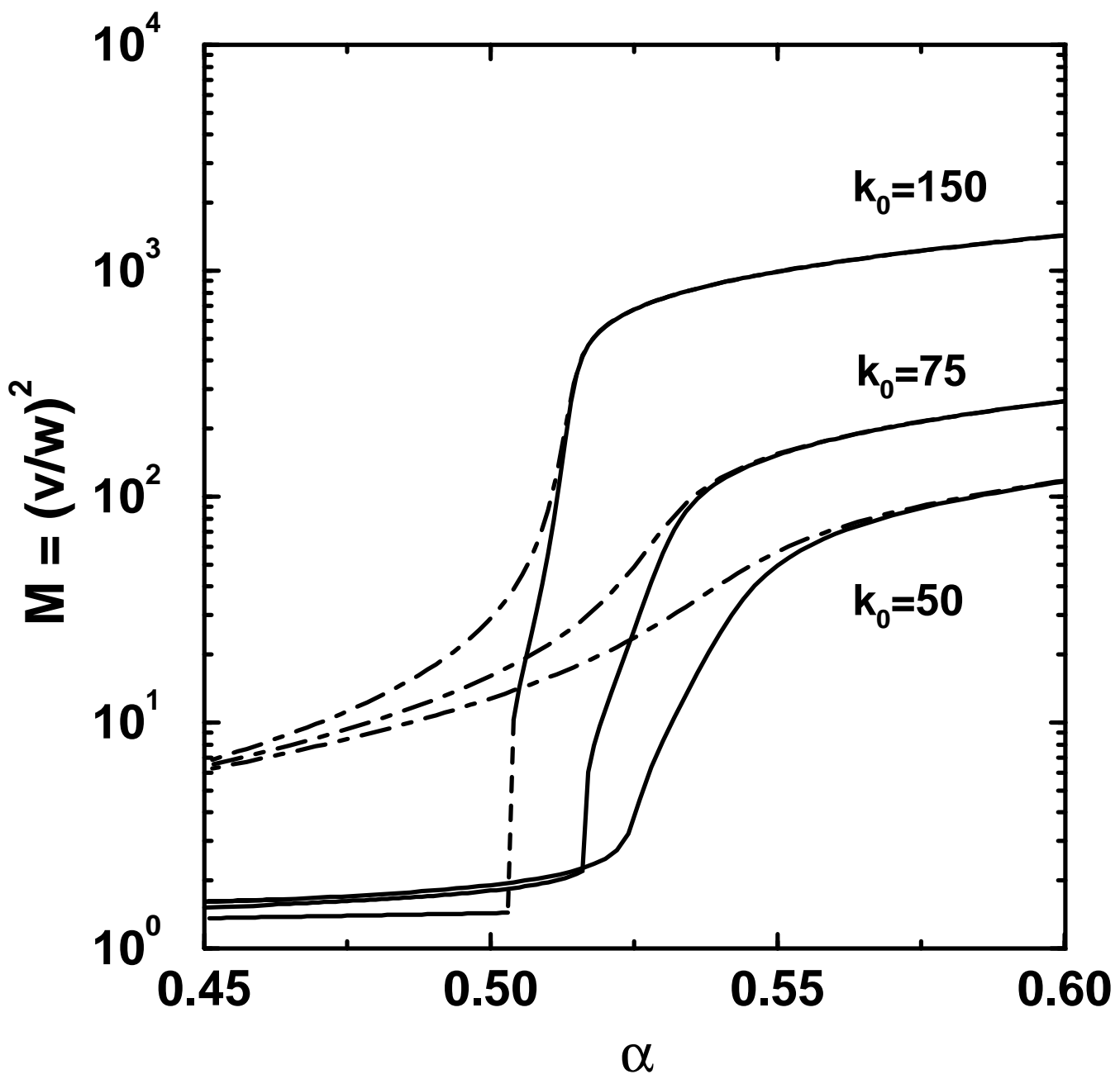




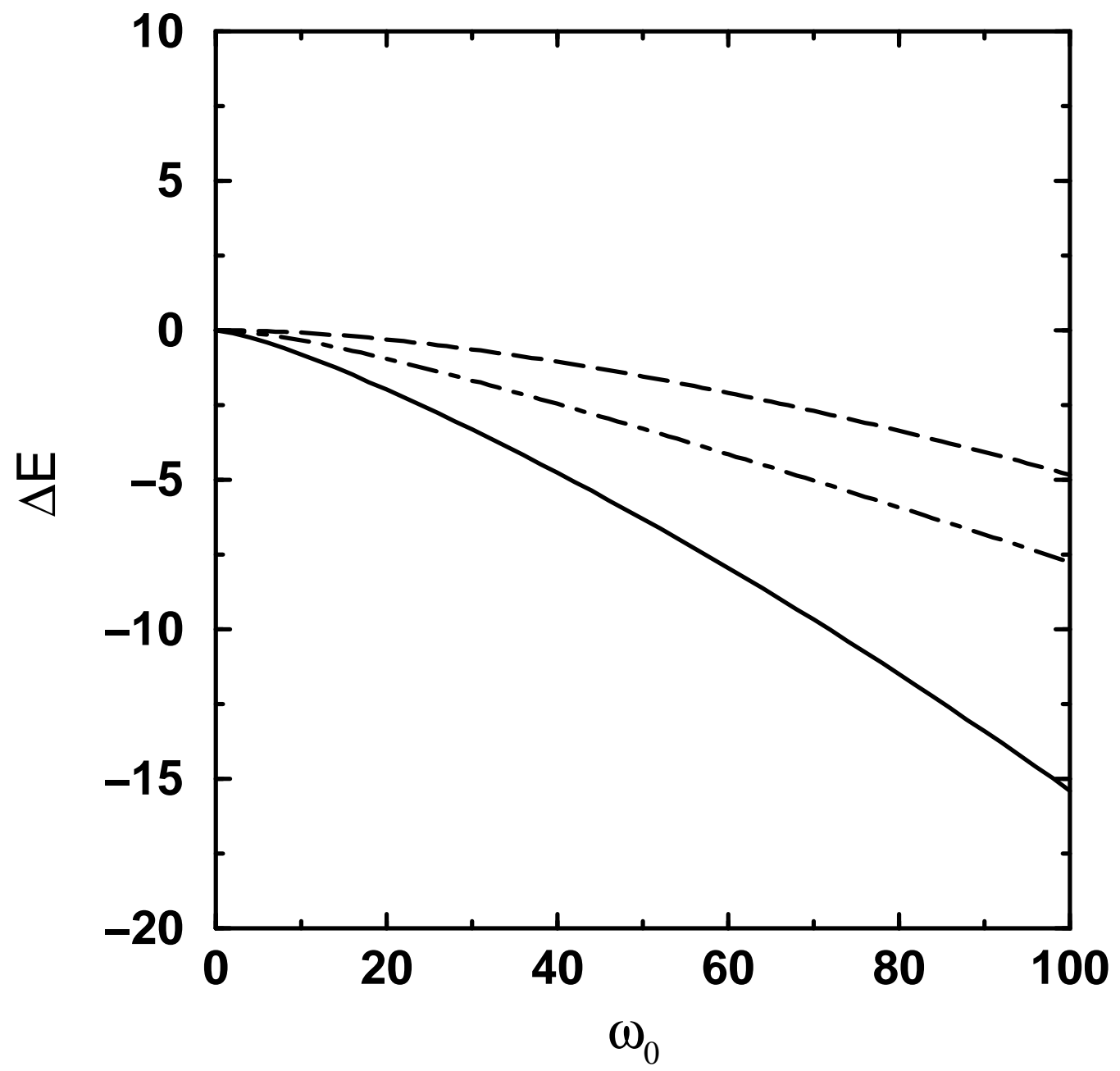




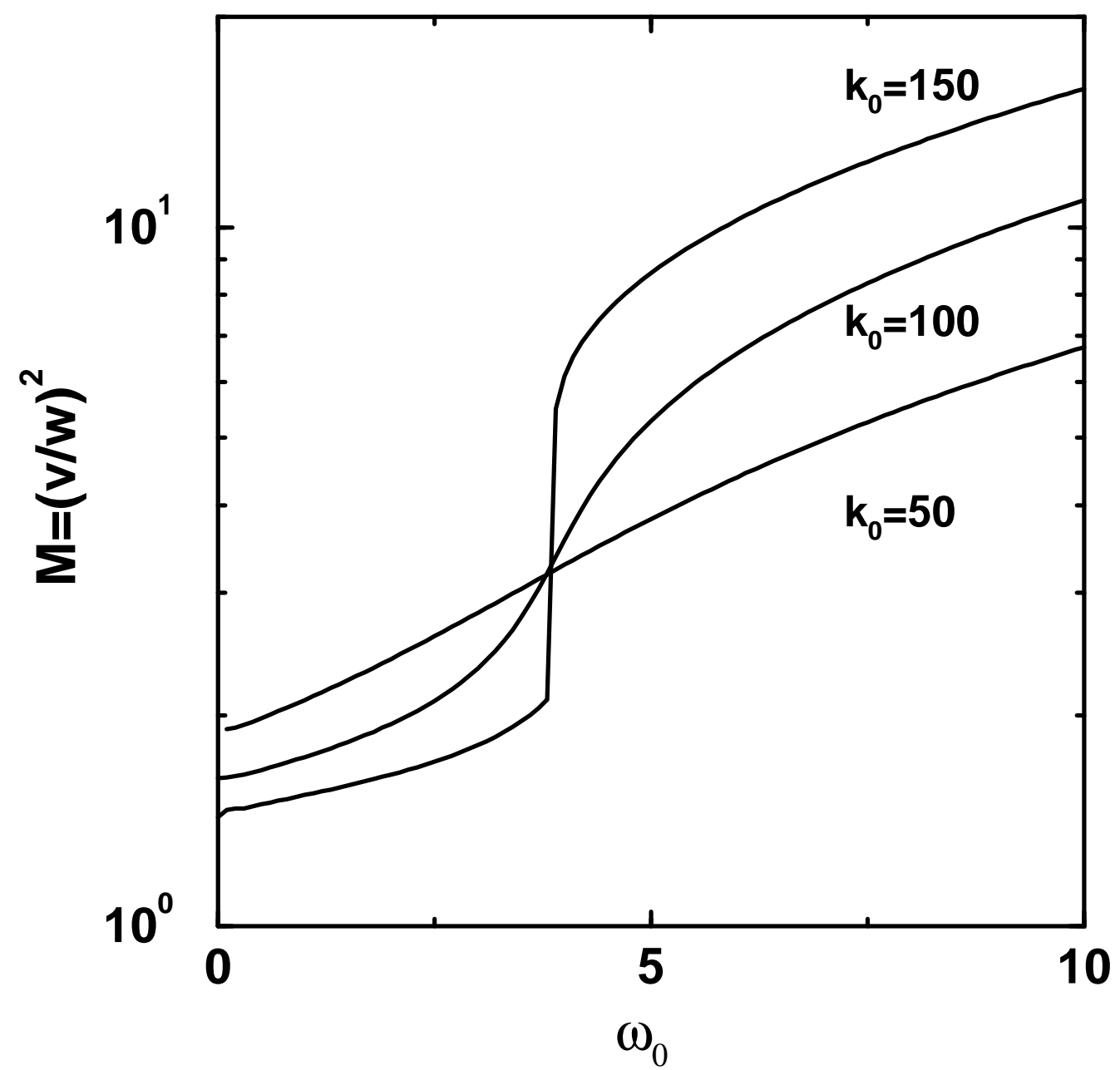

